\title{
The effects of inescapable shock on the retention of a previously learned response in an appetitive situation with delay of reinforcement
}

\author{
RICHARD S. CALEF, MICHAEL C. CHOBAN, JIM P. SHAVER, and JACK D. DYE \\ West Virginia Wesleyan College, Buckhannon, West Virginia \\ and \\ E. SCOTT GELLER \\ Virginia Polytechnic Institute and State University, Blacksburg, Virginia
}

\begin{abstract}
During Phase 1 of this experiment, all rats received a delay of (food) reward in a straight alley. During Phase 2, rats received escapable, inescapable, or no footshock in an operant chamber. The results, which supported a motivational interpretation of learned helplessness, showed that only inescapable shock reduced speed in a response previously attained (running in the straight alley; Phase 3).
\end{abstract}

Dogs or rats exposed to inescapable (uncontrollable) shock have performed worse with later escape learning (test situation) than have subjects receiving an equivalent amount of prior escapable shock or no shock. This phenomenon has been called "learned helplessness" (e.g., Maier, Albin, \& Testa, 1973; Overmier \& Seligman, 1967; Seligman \& Beagley, 1975). The learned helplessness effect also has been shown when the reward used in the test situation is appetitive rather than aversive. In other words, deficits in the acquisition of an instrumental response to obtain a food reinforcer have been demonstrated following prior exposure to inescapable shock (Caspy \& Lubow, 1981; Rosellini \& DeCola, 1981; Rosellini, DeCola, \& Shapiro, 1982).

It should be noted that all of the previous learned helplessness studies investigated the effects of inescapable shock on the learning of a new response. Little research has focused on the effects of inescapable shock on a previously learned response. Hence, the main purpose of the present study was to investigate the effects of uncontrollable shock on the retention of a previously learned response.

The second purpose of the present research was to shed light on the theoretical mechanisms contributing to learned helplessness. Seligman (1975) theorized that learned helplessness develops when there is an expectation that an outcome is independent of responding. According to Maier and Seligman (1976), the expectancy that behavioral and environmental consequences are independent produces interference with the acquisition of an instrumental response to obtain food or escape from shock because of an associational and/or motivational deficit. That is, Maier and

Please address correspondence to R. S. Calef, Box 12, Department of Psychology, West Virginia Wesleyan College, Buckhannon, WV 26201.
Seligman postulated that perception of uncontrollability during learning causes deficits in responding during learning in the test situation because of motivational (i.e., lower response initiation) and/or associational (i.e., interference with subsequent learning of response contingencies) mechanisms.

In the previous appetitive studies, there was no way of determining whether motivational mechanisms, independent of associational mechanisms, were sufficient to produce the learned helplessness effect. Hence, in the present study, we attempted to determine whether motivational deficits independent of associational deficits are sufficient for inescapable shock to produce learned helplessness in an appetitive situation.

Subjects were first taught in Phase 1 to run down a straight runway for a food reward that was delayed for $15 \mathrm{sec}$. The rats were then given escapable, inescapable, or no shock during Phase 2. During Phase 3, all rats were tested in the runway, again for a delayed food reward. Delay of reinforcement was used to decrease the probability that reduced motivation produced by inescapable shock would be precluded by the immediate food reward presented during pre- and posttesting periods.

If a motivational deficit independent of an associational deficit is sufficient for inescapable shock to produce learned helplessness in an appetitive situation, animals given inescapable shock during Phase 2 should run slower when tested for a previously learned response than should animals given escapable or no shock during Phase 2. If inescapable shock produces learned helplessness in an appetitive situation because of only associational deficits, subjects first taught to run down a runway for delayed reward, then given inescapable shock, and subsequently tested in the runway should not run slower than animals given escapable or no shock. We wanted to find out whether inescapable shock can produce an associational 
deficit in a response that has already been acquired and presumably has attained maximum association.

\section{METHOD}

\section{Subjects}

The subjects were 33 experimentally naive male albino rats of the Sprague-Dawley strain; they were approximately 90 days old at the beginning of the experiment. The animals were acquired from Dominion Laboratories, Dublin, Virginia, where they had been bred and reared. They were maintained on ad-lib water and run during the light phase of the 12-h-dark/12-h-light housing schedule.

\begin{abstract}
Apparatus
A straight-alley food runway and two operant chambers were used. The alley was composed of a $33-\mathrm{cm}$ gray startbox, a $66-\mathrm{cm}$ black runway section, and a $33.5-\mathrm{cm}$ black goalbox. The inner width and height of each section of the runway were 12.5 and $15.6 \mathrm{~cm}$, respectively. The walls and floor of the runway were constructed of plywood. Photocell and clock circuitry provided measures of traversal time over the last $30.5-\mathrm{cm}$ (1-ft) segment of the startbox (start measure), the first 30.5$\mathrm{cm}$ segment of the runway (run measure), and the last $30.5-\mathrm{cm}$ segment of the runway (goal measure).

The operant chambers were used either to administer the shock-escape training or to house the no-shock control animals during each treatment session. The chambers were $20.5 \mathrm{~cm}$ wide $\times 30 \mathrm{~cm}$ long. The long sides of the chambers were constructed of clear Plexiglas, the other walls of stainless steel, and the floor of 32 stainless steel grids, $5 \mathrm{~mm}$ in diameter and spaced $.5 \mathrm{~mm}$ apart. Between Grids 16 and 17, a wooden barrier separated the shocking operant chamber into two segments. The wooden ceiling was $10.2 \mathrm{~cm}$ high. The wooden ceiling could be opened on the left or right side, independent of each other. A Grason Stadler scrambler shock source (Model 1064-GS) provided shock to the grid floor. The nonshock operant chamber was essentially the same as the shocking chamber, except that no shock was provided to the grid floor.
\end{abstract}

\section{Procedure}

Fourteen days prior to the first day of training (Day 15), all rats were placed on a 23-h food-deprivation schedule, which was maintained throughout the study. On Days 7-14, the rats were taken from their individual home cages and handled for about 2 min each. On Days 13 and 14 , the rats were taken to the experimental laboratory, where they were allowed to explore the start and run sections of the runway apparatus while the clocks, photocells, and doors were operated. On those days, approximately $1 \mathrm{~g}$ of 45-mg Noyes pellets, identical to the subsequent reinforcement pellets, was incorporated into the rats' daily food rations.

During Phase 1, all animals were given 42 trials ( 3 trials per day) of continuous reinforcement training (four pellets) for 14 days in the straight runway with a $15-\mathrm{sec}$ delay of reward. During this time, the goal cup (containing four pellets) was slid under an opening in the goalbox subsequent to $15 \mathrm{sec}$ of confinement of the rat in the goal compartment. Following Phase 1, the subjects were matched in triads according to their start speeds and then randomly assigned to one of three groups (11 rats per group).

During Phase 2 of shock-escape training, a yoked-control design was employed in which Group CS (contingent or controllable shock) was shaped to barpress to turn off shock. Escape training consisted of exposing animals in Group CS to 350 trials (50 per day for 7 days) of up to $30 \mathrm{sec}$ of $1-\mathrm{mA}$ shock, which could be escaped by pressing the bar three times (FR3). On the first 5 trials, the lever was locked and each animal received the full $30 \mathrm{sec}$ of shock. On Trials 6-30, if the animal did not fulfill the FR3 contingency within the first $20 \mathrm{sec}$ of shock presentation, it was dragged onto the bar by the experimenter until the schedule was met. On Trials 31-350, the animal was left alone to perform the FR3 contingency. This procedure produced reliable escape in all rats. Each animal in Group CS was yoked to an animal in Group NCS (noncontingent, or noncontrollable shock) so that each pair received iden- tical shock durations and schedules. The animal in Group CS was placed in the left compartment of the operant shock chamber, and the partner from Group NCS was placed in the right compartment with no lever. Shock was therefore inescapable for the Group NCS animal, since its termination was controlled by the rat in Group CS. The yoked NCS animals were dragged when their Group CS partners were dragged; however, escaping shock was dependent on the performance of the Group CS animal.

Animals in Group NS (no shock) were placed in a segment of a noshock operant chamber similar in appearance and structure to that of the shock operant chamber, except these animals received no shock and no dragging. The Group NS member of each triad was placed in the no-shock chamber during the same time of day that the Group CS and NCS triad partners were treated. During each day, a barpress by Group CS animals produced alternating $30 \mathrm{sec}$ and $15 \mathrm{sec}$ of relief with every 10 shocks. In Phase 3 , all subjects were given the same runway treatment that they had in Phase 1, except that this period lasted for 4 days rather than 14 .

During Phases 1 and 3, trials were administered to three squads. Squads 1 and 2 consisted of four triads each, whereas Squad 3 consisted of three triads. The running order of subjects within a squad was randomized from day to day. The intertrial interval was approximately $20 \mathrm{~min}$, since a subject did not receive the second daily trial until all subjects in the squad had completed the first trial.

The running trials during Phases 1 and 3 were initiated by placing the rat backwards in the startbox. Placement of the rat in the startbox initiated the clock; the rat's turning around stopped the first clock and started the second clock. Thus, start time was essentially a measure of how long it took the animal to turn around. Movement down the alley terminated Clock 2 (run time) and started Clock 3; further movement toward the goalbox turned off Clock 3 (goal time). Following the rat's entry into the goalbox, a retrace door was dropped. Subsequent to consumption of the pellets, the rat was removed from the apparatus to a nearby carrying cage to await the next trial.

\section{RESULTS}

Since the start, run, and goal measures showed similar findings, and the goal measure is most relevant to a motivational interpretation, only the goal data are presented. The goal latencies were converted to reciprocals to yield goal speeds. Figure 1 illustrates the group mean speeds as a function of days for Groups NCS, CS, and NS during the last 2 days of pretraining and the 4 days of posttraining.

As depicted in Figure 1, all groups were running at approximately the same speed during the terminal pretraining phase. A 3 (groups) $\times 2$ (days) analysis of variance (ANOVA) for the last 2 days of terminal acquisition yielded no significance $(p>.05)$.

The posttraining data of Figure 1 shows that Group NCS ran markedly slower than Groups CS and NS throughout the posttraining phase, whereas Groups CS and NS demonstrated equivalent running speeds. A 3 (groups) $\times 4$ (days) ANOVA was performed on the 4 days of posttraining data. The analysis yielded only a significant main effect of groups $[F(2,20)=19.50$, $p<.001]$. Tukey paired comparisons between the groups' mean goal speeds showed that Group NCS ran significantly slower than did Groups CS and NS during the posttraining phase $(p<.001)$, but that Groups CS and NS did not exhibit significantly different speeds $(p>.05)$. 


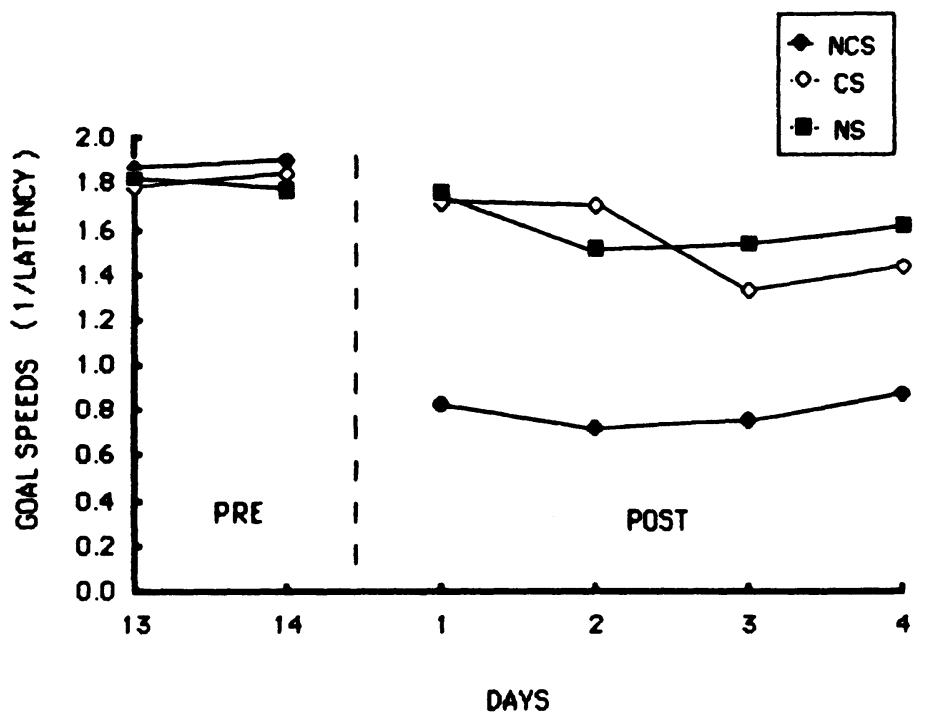

Figure 1. Group mean goal speeds as a function of days during the last 2 days of pretraining and during posttraining for Groups NCS, CS, and NS.

\section{DISCUSSION}

The results suggest that inescapable shock not only impairs the learning of new responses (as shown in previous studies) but also produces deficits in the performance of previously learned behaviors. If this finding can be obtained in human studies with further research, then this learned helplessness retention effect may provide a more valid and applicable model for human depression, since human depression usually involves an impairment of previously learned behavior.

In addition, the present findings also support the contention that a motivational deficit independent of an associational deficit is sufficient for inescapable shock to produce learned helplessness in an appetitive situation. In other words, the fact that animals first taught to run down a runway for delay of reward and then given inescapable shock turned around and ran slower down the runway than did animals given no shock or controllable shock, strongly suggests that the slower speeds during Phase 3 were primarily due to motivational rather than associational deficits. The fact that Group NCS showed slower speeds than the other groups in the goal measure particularly supports a motivational interpretation of the learned helplessness effect, since most motivational research shows the greatest differences in the goal measure (e.g., shift in magnitude of reward studies; Black, 1968).

It should also be noted that the present data do not support the results from studies investigating whether learned helplessness can be explained simply on the basis of inescapable shock's producing decrement in the motivation to initiate responding in an appetitive situation. In particular, Rapaport and Maier (1978) and Rosellini (1978) have reported that prior exposure to inescapable shock does not affect measures such as latency to reach for or ingest food. Similarly, Plonsky, Warren, and Rosellini (1984) showed no impact of inescapable shock on polydipsia. The many methodological differences (e.g., footshock vs. tail shock; delayed vs. immediate reward; learned vs. instinctual dependent variables) between the present study and the previous experiments could certainly account for the discrepancy in the findings. Further research should investigate which particular variable(s) account for the discrepancy.

It is noteworthy that alternative, nonmotivational conceptions can explain the present findings. For example, inescapable shock may have reduced speeds in Group NCS during Phase 3 because the uncontrollability of shocks may have produced forgetting, may have caused the subjects to learn an incompatible freezing response that may have generalized to the alley, or may have caused the unconditioning of the running response.
Nevertheless, the present findings do contribute to an understanding of the possible mechanisms contributing to the learned helplessness effect. The present data also conflict with that of Chen and Amsel (1977), who found faster speeds in the runway for rats receiving inescapable shock than for rats receiving no shock. Again, the variety of methodological differences between the studies (i.e., footshock vs. tail shock; delayed vs. immediate reward; a previously learned response vs. a new response serving as the dependent variable) may have accounted for the discrepancies, and it remains for further research to determine which variables may have contributed to the discrepancy.

Finally, the generality of the learned helplessness effect was questioned when Beatty (1979) found no deficit in barpressing to escape shock at any duration of inescapable footshock. Beatty's (1979) failure to obtain learned helplessness may be explained by the hypothesis that animals receiving inescapable footshock may in fact be receiving contingent shock, since rats can escape footshock by standing up on their hind feet if both feet happen to be located on the same grid. The present study attempted to alleviate this problem by lowering the ceiling of the shock chamber and using twice the number of closely spaced grids. These changes to the apparatus were designed to decrease the probability that the NCS group would actually receive contingent shock. It would be instructive to replicate Beatty's (1979) study using the foot-shock modification of the present study. With this modification in the apparatus, one would expect inescapable footshock to produce an impairment in barpressing to escape shock.

\section{REFERENCES}

BEATTY, W. W. (1979). Failure to observe learned helplessness in rats exposed to inescapable footshock. Bulletin of the Psychonomic Society, 13, 272-273.

BLACK, R. W. (1968). Shifts in magnitude of reward and contrast effects in instrumental and selective learning: A reinterpretation. Psychological Review, 75, 114-126.

CASPY, T., \& LUBOW, R. E. (1981). Generality of US preexposure effects: Transfer from food to shock or shock to food with and without the same response requirements. Animal Learning \& Behavior, 9 , 524-532.

Chen, J. S., \& AMSel, A. (1977). Prolonged, unsignaled, inescapable shocks increase persistence in subsequent appetitive instrumental learning. Animal Learning \& Behavior, 5, 377-385. 
Maier, S. F., Albin, R. W., \& Testa, T. J. (1973). Failure to learn to escape in rats previously exposed to inescapable shock depends on the nature of the escape response. Journal of Comparative \& Physiological Psychology, 85, 581-592.

Maier, S. F., \& Seligman, M. E. P. (1976). Learned helplessness: Theory and evidence. Journal of Experimental Psychology: General, $105,3-46$.

Overmier, J. B., \& Seligman, M. E. P. (1967). Effects of inescapable shock upon subsequent escape and avoidance learning. Journal of Comparative \& Physiological Psychology, 63, 23-33.

Plonsky, M., Warren, D. A., \& Rosellini, R. A. (1984). The effects of inescapable shock on appetitive motivation. Bulletin of the Psychonomic Society, 22, 229-231.

RAPAPORT, P. M., \& MAIER, S. F. (1978). Inescapable shock and foodcompetition dominance in rats. Animal Learning \& Behavior, 6, 160-165.
Rosellini, R. A. (1978). Inescapable shock interferes with the acquisition of a free appetitive operant. Animal Learning \& Behavior, 6, 155-159.

Rosellini, R. A., \& DeCola, J. P. (1981). Inescapable shock interferes with the acquisition of a low-activity response in an appetitive context. Animal Learning \& Behavior, 9, 487-490.

Rosellini, R. A., DeCola, J. P., \& Shapiro, N. R. (1982). Crossmotivational effects of inescapable shock are associative in nature. Journal of Experimental Psychology: Animal Behavior Processes, 8, 376-388.

Seligman, M. E. P. (1975). Helplessness. San Francisco: Freeman. Seligman, M. E. P., \& Beagley, G. (1975). Learned helplessness in the rat. Journal of Comparative \& Physiological Psychology, 88, 534-542.

(Manuscript received for publication September 28, 1985.) 